\title{
Caracterización socio-epidemiológica y evolución de la tuberculosis en la Región Metropolitana de Chile, 2005 a 2018
}

\author{
Socio-epidemiological caracterization and evolution of tuberculosis in the Metropolitan \\ Region of Chile, 2005 to 2018
}

Claudio Olmos ${ }^{1,2}$, Valeria Stuardo 3 , Paulina Ramonda ${ }^{4}$ y Carlos Peña 5

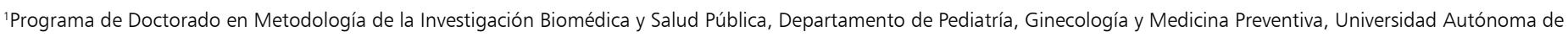
Barcelona. Barcelona, España.

${ }^{2}$ Escuela de Medicina Universidad Andrés Bello. Santiago, Chile.

${ }^{3}$ Programa de Epidemiología, Escuela de Salud Pública, Universidad de Chile. Santiago, Chile.

${ }^{4}$ Programa de Tuberculosis Servicio de Salud Metropolitano Sur, Hospital Barros Luco Trudeau. Santiago, Chile.

${ }_{5}^{5}$ rograma de Tuberculosis Servicio de Salud Metropolitano Central, Hospital San Borja Arriarán. Santiago, Chile.

Financiamiento: No hubo financiamiento externo.

Conflicto de intereses: Los autores declaran la ausencia de conflictos de interés.
\end{abstract}

Recibido: 26 de noviembre de 2019 / Aceptado: 20 de mayo de 2020

\section{Resumen}

Introducción: El Programa chileno de Control y Eliminación de Tuberculosis (PROCET) ha permitido reducir la mortalidad por esta enfermedad en 78\%, entre los años 2004 y 2013. No obstante, luego de décadas de resultados exitosos, a partir del 2000 ocurrió un enlentecimiento en la reducción de incidencia y desde el 2014, un aumento de ésta. Objetivos: Describir la evolución socioepidemiológica de los casos de tuberculosis (TBC), desde 2005 al 2018, estratificando según país de origen, en Servicios de Salud de la Región Metropolitana (RM). Métodos: Estudio transversal con componente ecológico. Se consideraron las variables: edad, sexo, localización de la TBC, confirmación bacteriológica, co-infección con VIH, privación de libertad, país de procedencia y efectividad de la terapia anti-tuberculosis. Resultados: Se notificaron 7.507 casos, de los cuales $75,1 \%$ fue de localización pulmonar y $65,4 \%$ bacteriológicamente demostrado. Un 19,0\% de los casos pertenecieron a personas no nacidas en Chile, con un aumento de esta proporción en los últimos seis años. Las tasas de incidencia, descendientes en la primera mitad del período, exhibieron un alza a partir del 2012, alejándolas del umbral de eliminación. Se observó en 74,3\% éxito en la terapia y $13,4 \%$ de muerte en los casos evaluados. Conclusiones: En los últimos años, la incidencia de TBC en la RM aumentó. Los nuevos grupos vulnerables, especialmente los migrantes, requieren con urgencia, que se implementen y refuercen estrategias como la educación, pesquisa activa y acciones de control de TBC.

Palabras clave: enfermedades infecciosas; incidencia; vigilancia epidemiológica; inmigrantes.

\begin{abstract}
Background: The Chilean Program for the Control and Elimination of Tuberculosis (PROCET) has reduced mortality from this disease by $78 \%$ between 2004 and 2013. However, after decades of successful results, starting in 2000 there was a slowdown in the reduction of incidence and since 2014, an increase in it. Aim: To describe the socioepidemiological evolution of tuberculosis (TB) cases treated at health clinics in the Metropolitan Region (MR) of Chile from 2005 to 2018, stratifying by country of origin. Methods: Cross-sectional study with ecological components, including analyses of age, sex, TB localization, bacteriological confirmation of diagnosis, co-infection with HIV, incarceration, country of origin, and effectiveness of tuberculosis treatment. Results: A total of 7,507 TB cases were recorded during the study period; $75.1 \%$ of cases were pulmonary tuberculosis, and $65.4 \%$ were bacteriologically confirmed. Overall, $19.0 \%$ of cases involved persons born outside of Chile, with the proportion of cases in foreignborn persons increasing over the past 6 years. Incidence decreased during the first half of the study period but then began to increase after 2012, moving the country away from the threshold of elimination. A total of $74.3 \%$ of cases were treated successfully, and $13.4 \%$ expired. Conclusion: In recent years, TB incidence has increased in the MR of Chile, possibly attributable to growing populations of vulnerable groups such as immigrants. This finding suggests an urgent need to implement and reinforce strategies such as education, an active screening model and more efficient contact tracing to prevent the spread of TB.

Keywords: infectious diseases; incidence; epidemiological surveillance; immigrants.
\end{abstract}

\section{Correspondencia a:}

Valeria Stuardo Ávila

valeria.stuardo@uchile.cl 


\author{
Introducción
}

$\mathrm{D}$ urante las últimas décadas, Chile ha sido el país de Sudamérica con menor incidencia de tuberculosis $(\mathrm{TBC})^{1}$, manteniéndose cercano a los 13 casos por 100.000 habitantes $^{2}$.

El Programa chileno de Control y Eliminación de Tuberculosis (PROCET), es una política pública que le ha permitido al país, reducir la mortalidad por esta enfermedad en $78 \%$, pasando de 5,8 a 1,3 muertes por 100.000 habitantes entre 2004 y 2013, así como reducir en $70 \%$ la incidencia, pasando de 46,5 casos a 12,4 por 100.000 habitantes entre 1990 y $2013^{1,3,4}$.

No obstante, luego de décadas de resultados exitosos, a partir del 2000 ocurrió un enlentecimiento en la reducción de incidencia y desde el 2014, un aumento de ésta, alcanzando los 14,7 x 100.000 en $2017^{2}$. Esta cifra sigue estando por debajo de la incidencia de la región; sin embargo, implica el primer retroceso sanitario desde la implementación del programa².

Publicaciones asocian el fenómeno del enlentecimiento en la reducción de incidencia a la aparición de nuevos grupos susceptibles, como la población extranjera ${ }^{5-11}$. La migración en sí, no es un factor de riesgo, pero puede llevar a las personas a situaciones que aumentan su riesgo de adquirir enfermedades ${ }^{11-13}$. Éstas presentarían escasa adherencia a la terapia, baja confianza con el equipo de salud, y su estigmatización las conduce a un aislamiento, convirtiéndolas en sujetos especialmente vulnerables ${ }^{14-16}$.

La evidencia indica que en aquellos países que alcanzan una baja incidencia de TBC, aumenta la importancia relativa de casos en personas extranjeras ${ }^{13-15}$, los que se manifiestan en personas con TBC que ingresan al país o que desarrollan la enfermedad a poco tiempo del arribo al país ${ }^{16-18}$.

En los últimos 30 años, Chile ha tenido un aumento sostenido de extranjeros que llegan al país, pasando de 83.000 migrantes en 1982, a 745.772 en el $2017^{19,20}$. El $65,3 \%$ de ellos se concentra en la Región Metropolitana (RM). Los seis países que aportan mayor proporción de personas al total de inmigrantes, provienen de Perú, Colombia, Venezuela, Bolivia, Argentina y Haití, constituyendo $77,6 \%$ de la población inmigrante internacional en Chile ${ }^{19-21}$; todos países con mayor incidencia de TBC que Chile'.

En suma, el último informe entregado por los países de baja incidencia de TBC de las Américas, define a los extranjeros como el grupo con mayor riesgo de adquirir la enfermedad en Chile ${ }^{22}$.

Por ello, este estudio se propone describir la evolución socio-epidemiológica de los casos de TBC, desde el año 2005 al 2018, estratificando según país de origen, en los principales Servicios de Salud (SS) de la RM.

\section{Métodos}

Se trata de un estudio descriptivo con componente ecológico, en el cual se analizaron los casos de TBC registrados por el PROCET en los Servicios de Salud Metropolitano Central (SSMC), Norte (SSMN) y Sur (SSMS), entre los años 2005 y 2018.

Para el SSMC y SSMS, se contó con la totalidad de los registros desde enero de 2005 a diciembre de 2018, mientras que, para el SSMN, sólo se dispuso de los registros a partir del 2012. La población cubierta por estos servicios fue de 3.429.872 personas en el 2018, lo que representa $48,2 \%$ de todos los habitantes de la RM y $19,5 \%$ de la población total del país.

Informes preliminares muestran que estos tres SS tienen altas tasas de TBC y reciben una importante cantidad de extranjeros. En efecto, $68 \%$ de los migrantes de la RM se concentra en este territorio, representando aproximadamente $9,8 \%$ del total de la población cubierta por los tres SS.

Se revisaron los casos diagnosticados y se recogieron los siguientes antecedentes provenientes del formulario de notificación obligatoria: sexo, edad, localización de la TBC (pulmonar y extra-pulmonar), confirmación bacteriológica (frotis, cultivo, reacción de polimerasa en cadena (RPC), y mediante otros hallazgos), co-infección con VIH, privación de libertad, comuna de residencia, país de procedencia y efectividad de la terapia anti-TBC.

Para la variable efectividad de la terapia anti-TBC se definieron cuatro atributos de acuerdo a los criterios que describe la OMS: "éxito global" del tratamiento si es que el paciente culmina su terapia; "abandono o pérdida de seguimiento" si registra inasistencia continua a la terapia por más de 30 días; "fracaso", cuando la baciloscopia persiste positiva hasta el cuarto mes de tratamiento, o si luego de hacerse negativa reaparece positiva durante la terapia y "muerte", si es que esta ocurre durante el tratamiento, independiente de la causa.

La información recolectada hizo posible la construcción de tasas de incidencia, estratificadas en dos grandes grupos: casos chilenos y casos extranjeros. Para la incidencia de TBC en personas nacidas en Chile, se utilizó como denominador, la población registrada en las comunas que abarcan los SS, mientras que la tasa en personas no nacidas en Chile, fue calculada, a partir de una aproximación basada en los registros de visas temporarias y permisos definitivos.

Se utilizó el paquete estadístico STATA 13.0 y el software Microsoft Excel 2016 para el análisis de datos. Se realizó un análisis de correlación lineal de Pearson entre la tasa de incidencia y los casos extranjeros. Se usaron pruebas de $\chi^{2}$ para analizar los resultados de la terapia. Se consideró estadísticamente significativo a un p valor menor a 0,05 . 
La tasa de TBC en casos chilenos, muestra una evolución similar a la anteriormente descrita para los tres SS, aunque más baja. Por el contrario, la tasa de incidencia en casos de personas no nacidas en Chile, se muestra más alta en todos los años a partir del 2006, con un fuerte incremento desde 2012, alcanzando tasas de hasta 72,2 x 100.000 hab. Los cambios en la tasa de incidencia de TBC para la población de estudio, así como su estratificación según nacionalidad, se observan en la Figura 1.

Durante el período estudiado se registraron 7.507 casos de TBC diagnosticados. El 65,8\% correspondió a hombres y la media de edad fue de 44,5 años (mediana de edad = mujeres: 46,8; hombres: 43,3 años).

Del total de casos, 75,1\% correspondió a TBC pulmonar (TBC-P) y 87\% de ellos fueron bacteriológicamente demostrados. El 24,9\% de los casos se describieron como TBC extra-pulmonar, destacándose las formas ganglionar y pleural como las más frecuentes.

Del mismo modo, se observó que, para todo el período, $12,1 \%$ de los casos correspondió a co-infección TBC/VIH, con un leve aumento a lo largo del período ( $0,2 \%$ anual).

Por otra parte, la mayoría de los casos en personas privadas de libertad, provino del SSMC, el que da cobertura al recinto penitenciario más grande del país. Estos casos representaron por sí solos, $14,3 \%$ de todos los casos de TBC notificados en este SS, aunque esta cifra descendió a 7\% el año 2018. Los SSMN y SSMS mostraron una baja proporción de casos en población penal (1,4 y 3,5\%, respectivamente).

De los 7.507 casos, 19,0\% perteneció a personas no nacidas en Chile. El $60 \%$ de ellos provino de Perú, $15 \%$ de Haití, $5 \%$ de Bolivia y $12 \%$ de otros países de América Latina. En su conjunto, los casos en personas provenientes de Perú y Haití, representaron 73,5\% de los casos en extranjeros notificados el 2018. En la Tabla 1 se observan las características demográficas y clínicas de los casos, según origen.

Durante los primeros años del período estudiado, la mortalidad por TBC se mantuvo relativamente constante cercana a 1,2 x 100.000 hab., pero a partir del 2011, se observó un leve y sostenido aumento, llegando a 1,7 x 100.000 hab. en el 2018, con un pico de 2,6 x 100.000 hab. en el año 2015.

La tasa de incidencia de TBC para los tres SS, pasó de 22,5 x 100 mil hab. en 2005 a 25,3 x 100 mil hab. en 2018. A excepción de un pico de frecuencia en el 2009 , se observó que la incidencia de TBC mostró un descenso entre los años 2005 y 2012, pero a partir de este último año, se experimentó un aumento que superó incluso, las cifras observadas al inicio del período. Dicho incremento, también coincide con un alza en la proporción de casos extranjeros, pasando de constituir $9,1 \%$ de los casos totales en el 2012, a 35,2\% en 2018. Al ser sometidas a un análisis de correlación simple, no se observa asociación entre ambas variables, aun excluyendo el año 2009, como un posible año de sobre-diagnóstico $(r=0,13 ; \mathrm{p}=0,67)$.

\begin{tabular}{|c|c|c|}
\hline \multirow{2}{*}{$\begin{array}{l}\text { Característica socio-demográfica y } \\
\text { clínica }\end{array}$} & \multicolumn{2}{|c|}{ Casos de tuberculosis } \\
\hline & Chilenos & Extranjeros \\
\hline \multicolumn{3}{|l|}{ Sexo $(\%)$} \\
\hline Femenino & 33,1 & 39,5 \\
\hline Masculino & 66,9 & 60,5 \\
\hline \multicolumn{3}{|l|}{ Edad (media en años) } \\
\hline Femenino & 50,7 & 32,7 \\
\hline Masculino & 45,5 & 33,1 \\
\hline Total & 47 & 33,4 \\
\hline \multicolumn{3}{|l|}{ Localización (\%) } \\
\hline Pulmonar & 75,3 & 74,5 \\
\hline Pulmonar BD* & 65,7 & 63,9 \\
\hline Extra-pulmonar & 24,7 & 25,5 \\
\hline \multicolumn{3}{|l|}{ Condición asociada (\%) } \\
\hline Co-infección TBCNIH & 12,1 & 11,7 \\
\hline Privación de libertad & 8,8 & 1,7 \\
\hline
\end{tabular}

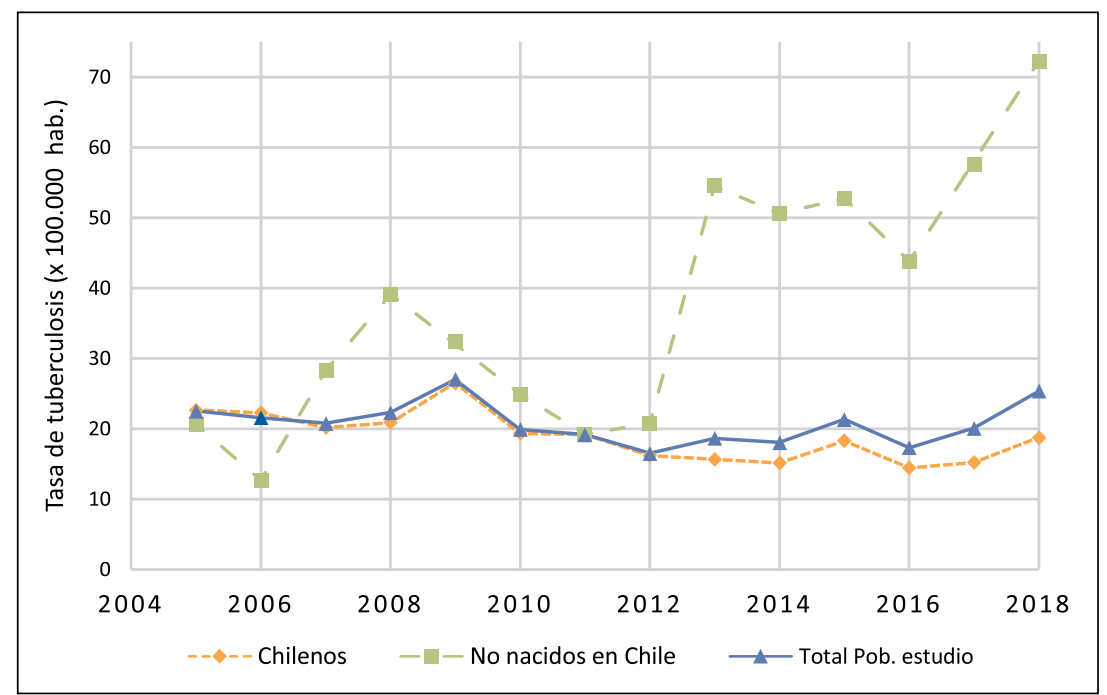

Figura 1. Tasa de incidencia de TBC en tres SS de la Región Metropolitana de Chile: incidencia de TBC en población chilena y población extranjera en Chile, durante el período 2005-2018. Fuente: Creación propia de los autores. 
Figura 2. Distribución y evolución de tasa de incidencia de TBC por 100 mil habitantes/año por comuna de la Región Metropolitana de Chile, entre el 2012 y 2018. Fuente: Creación propia de los autores.

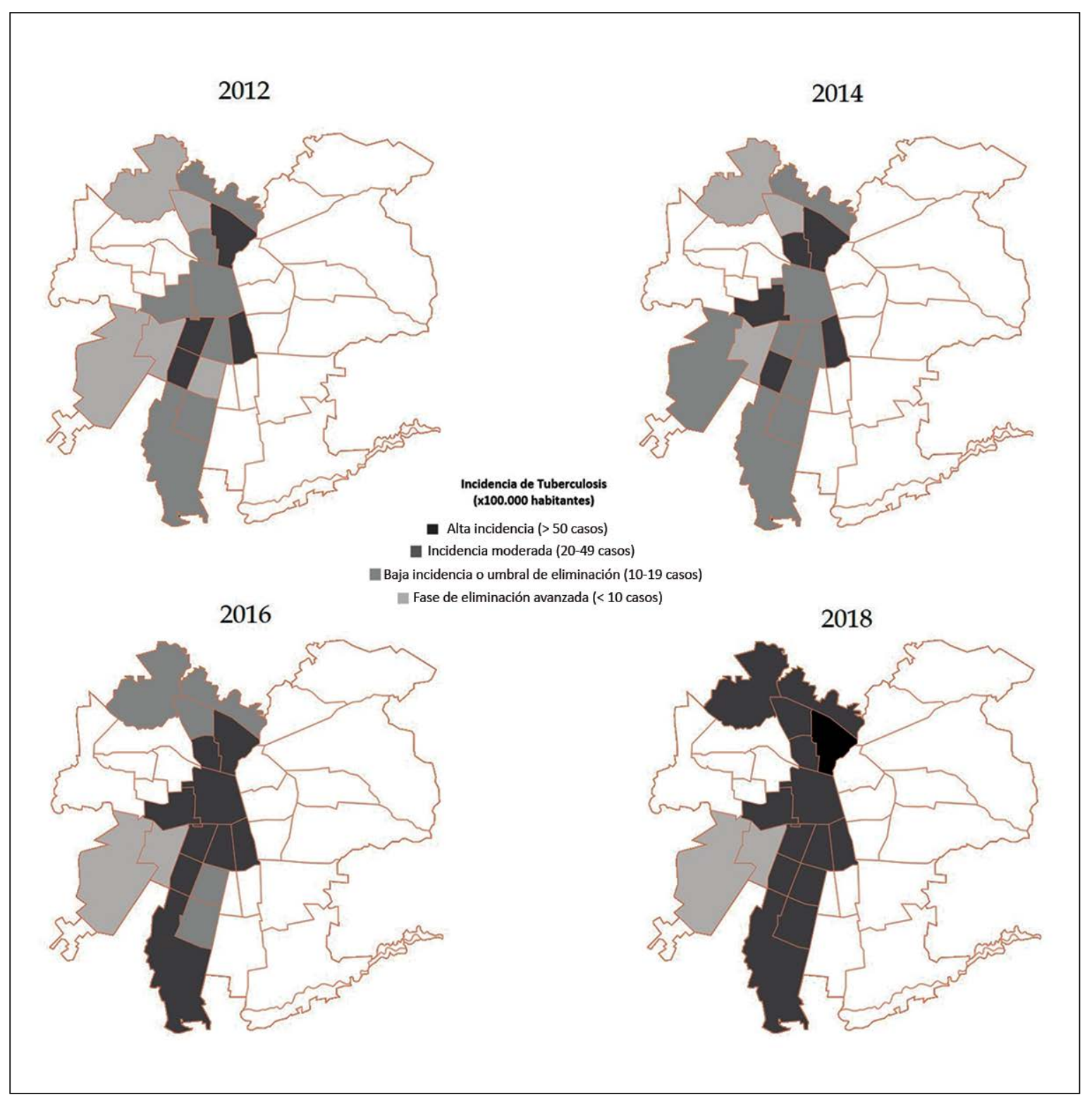

Las comunas que presentaron tasas más altas de TBC durante el período, fueron Santiago, San Joaquín, Lo Espejo, Pedro Aguirre Cerda, Independencia, Estación Central y Recoleta; esta última con más de 50 casos x 100.000 hab. en el 2018. La distribución de tasa de incidencia de TBC por comuna, entre el 2012 y 2018, se observa en la Figura 2.

En relación a los resultados de la terapia, sólo fueron considerados 4.394 casos del total, excluyendo aquellos casos con registros incompletos, así como los casos ocurridos durante el año 2018, muchos de los cuales aún no completaban su tratamiento al iniciar esta investigación. Del total de casos evaluados, se observó $74,3 \%$ de éxito, $12,1 \%$ de abandono, $13,4 \%$ de muerte y $0,1 \%$ de fracaso. Al observar estas cifras según nacionalidad, los extranjeros tienen mayor frecuencia de éxito en la terapia y menor porcentaje de muertes, pero mayor porcentaje de abandono en comparación a los chilenos. Estas diferencias resultaron ser estadísticamente significativas $(\mathrm{p}<0,001)$. Los resultados de la terapia anti-TBC, tanto en casos chilenos como en extranjeros, se muestran en las Figuras 3 y 4 , respectivamente. 


\section{Discusión}

La población de este estudio representa casi $20 \%$ de la población nacional, y concentra $25,9 \%$ de todos los casos notificados en el país durante los últimos cinco años ${ }^{2}$.

Al caracterizar los casos analizados, se observa que la TBC afecta principalmente a hombres cercanos a los 45 años, y a edades más tempranas en los casos extranjeros. La TBC-P concentró la mayoría de los casos $(75,1 \%)$ y un total de $65,4 \%$ fueron bacteriológicamente demostrados.

Las cifras de casos en personas privadas de libertad en el SSMC, están por sobre la proporción nacional ${ }^{2}$. Esto se explica porque ahí se encuentra uno de los recintos penitenciarios más grandes del país, y es sabido que dicha población tiene un riesgo de contagio 18 veces mayor al de la población general ${ }^{25}$.

Asimismo, la co-infección TBC/VIH implica un riesgo de letalidad tres veces mayor que la infección por TBC cuando no existe la co-morbilidad ${ }^{26}$. Según el último reporte global de TBC, $13 \%$ de todos los casos de TBC está co-infectado con VIH, lo que representaría sólo $46 \%$ del número de casos esperados ${ }^{27}$, poniendo en evidencia el sub-diagnóstico de esta condición. En este estudio, la proporción de casos de co-infección TBC/ VIH fue de $12,1 \%$, con tendencia al alza, y que se espera sea aún mayor, dadas las cifras que muestran a Chile como el país que reportó el mayor aumento de casos de infección por VIH (34\%), en los últimos cinco años en América latina ${ }^{28}$.

También, este estudio revela que desde el 2012 al 2018, la tasa de incidencia de TBC en los tres SS estudiados pasó de 16,5 a 25,3 x 100 mil hab. Estas cifras contribuyen en gran medida, al aumento en la tasa de incidencia de TBC a nivel nacional, la que aumentó de 12,5 en 2012 a 14,9 x 100 mil hab. en $2018^{2}$.

Del mismo modo, se ha observado un aumento de casos de personas no nacidas en Chile, las que provienen principalmente de Perú y Haití; ambos países con altas tasas de TBC (116,4 y 184,4 x 100 mil hab., respectivamente $)^{26}$. El porcentaje de casos extranjeros en los tres SS aumentó en $258 \%$. Esta situación coincide con el aumento de la población migrante en el país, que según cifras oficiales habría aumentado en $240 \%$ durante el mismo periodo ${ }^{19,21}$.

La evidencia científica sugiere que la principal causa de la diferencia entre la tasa de incidencia de TBC en casos autóctonos y extranjeros sería la reactivación de TBC latente ${ }^{14}$. Se han descrito diferencias en cuanto al riesgo de exposición a patógenos, vulnerabilidad a infecciones, vacunación, susceptibilidad genética, deficiencias nutricionales, co-morbilidades, estatus socio-económico, creencias culturales acerca de la TBC y barreras de acceso a la salud ${ }^{12,29,30}$.

En cuanto a la terapia anti-TBC, los deficientes resultados y la creciente mortalidad pueden explicarse desde

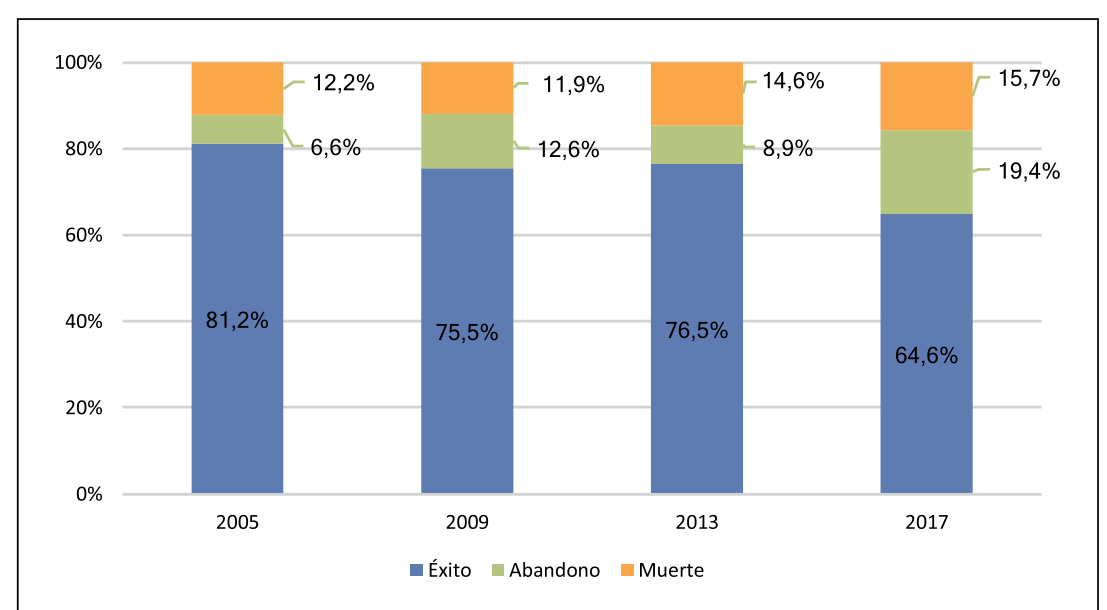

Figura 3. Resultados de la terapia anti-TBC en casos chilenos, durante los años 2005, 2009, 2013 y 2017. Fuente: Creación propia de los autores.

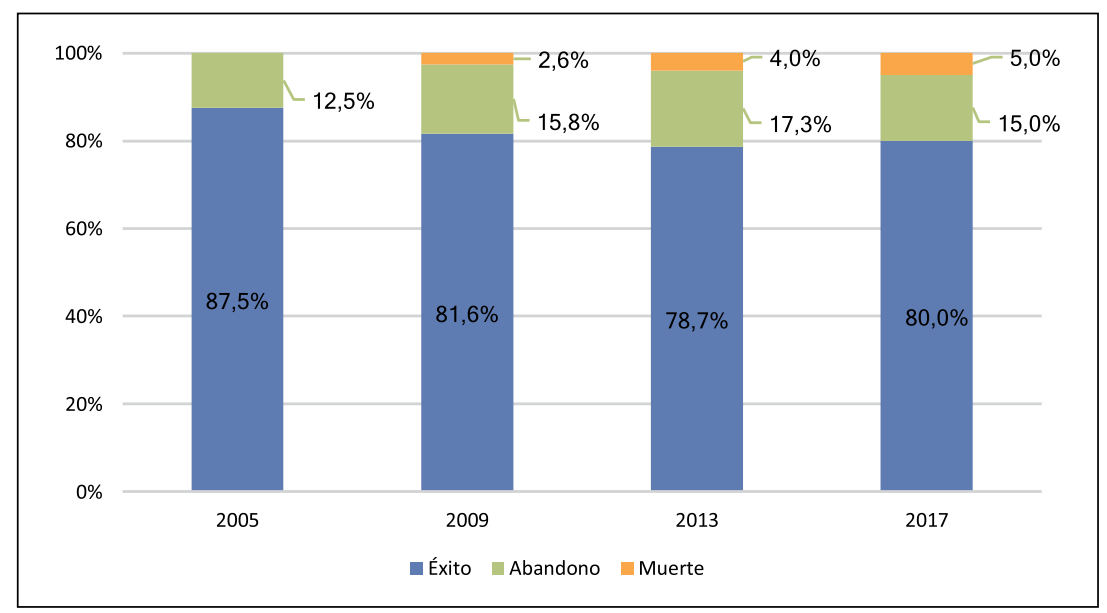

Figura 4. Resultados de la terapia anti-TBC en casos extranjeros de, durante los años 2005, 2009, 2013 y 2017 . Fuente: Creación propia de los autores.

dos aristas: por un lado, las condiciones de vida, la precariedad de la vivienda, co-morbilidades, abuso de drogas y desinformación de algunas personas ${ }^{31,32}$; y por otro lado, la ejecución de métodos de pesquisa poco efectivos para el contexto, estudio de contacto insuficiente, diagnóstico tardío y una creciente resistencia a farmacológica ${ }^{27,33}$.

En ese sentido, una fortaleza del PROCET, radica en que sus acciones de diagnóstico y tratamiento son gratuitas, tanto para chilenos como para extranjeros ${ }^{4}$; sin embargo, esta característica pierde su sentido si las personas más vulnerables no acceden a los SS.

Por ello, la estrategia de pesquisa pasiva que mayoritariamente ocupan los SS en Chile, resulta inapropiada para el nuevo escenario. Por el contrario, un modelo basado en la pesquisa activa permitiría realizar diagnósticos oportunos, disminuyendo las posibilidades de contagio. 
La implementación de agentes comunitarios en salud, trabajando coordinadamente entre las comunidades y los servicios de atención sanitaria, pareciera haber brindado mejores resultados a regiones con alta tasa de inmigrantes ${ }^{34,35}$. Asimismo, se hace necesario incorporar organizaciones de la sociedad civil (OSC) en la implementación de acciones de prevención y atención. Para ello, se recomienda: realizar un diagnóstico inicial de las comunidades; mayor eficiencia administrativa para mejorar el uso de sus recursos; ampliar las acciones de sensibilización hacia los gobiernos en la importancia de trabajar coordinadamente con la comunidad y formalizar los vínculos de colaboración con los mismos

Se recomienda, además, ampliar el estudio de contacto de los casos pulmonares bacilíferos en extranjeros, así como generar nuevas estrategias de prevención, considerando, por ejemplo, la terapia de TBC latente en migrantes provenientes de países de alta incidencia y personas inmunosuprimidas.

Algunas limitaciones de este estudio fueron la existencia de algunos registros incompletos, como es el caso de los resultados de la terapia y el hecho de que sólo se contó con registros del SSMN a partir del 2012. Razones logísticas para el desarrollo de este estudio nos llevaron a no incluir la casuística de los SSM Oriente y SS Suroriente. Por otro lado, el haber podido analizar la situación y evolución epidemiológica de algunas de las comunas con mayor incidencia de TBC en Chile, constituye una importante fortaleza. Los investigadores estiman que haber abarcado una población que representa casi $20 \%$ de la población nacional, y concentra el $25,9 \%$ de todos los casos notificados en el país durante los últimos 5 años entrega un panorama que refleja la situación epidemiológica de TBC en nuestro país.

\section{Conclusiones}

Así, los resultados de este estudio permiten concluir que existe un retroceso sanitario relacionado al manejo de la TBC en la región, alejando a Chile, del umbral de eliminación. Las crecientes tasas de incidencia, junto con la mayor proporción de casos en residentes nacidos en el extranjero, sugieren una necesidad urgente de acción por parte de las autoridades de salud, incluyendo el desarrollo y refuerzo de estrategias como la educación, un modelo de detección activa y un seguimiento de contactos más eficiente para prevenir la propagación de la TBC.

\section{Referencias bibliográficas}

1.- Organización Panamericana de la Salud. Situación de control de la tuberculósis en las Américas. 2018; https://iris.paho.org/bitstream/ handle/10665.2/49510/OPSCDE18036 spa? sequence $=2 \&$ isAllowed $=\mathrm{y}$

2.- Ministerio de Salud de Chile. Informe de situación epidemiológica y operacional del Programa Nacional de Tuberculosis 2018. 2019.

3.- Organización Panamericana de la Salud. Informe VII Reunión Regional de países de baja incidencia de tuberculosis de las Américas. Santiago-Chile 4 y 5 de julio de 2016.

4.- Ministerio de Salud de Chile. Programa Nacional para el Control y la Elimininacion de la Tuberculosis. Normas técnicas para el control y la eliminacion de la tuberculosis. 2014; 1-58. https://www.minsal.cl/sites/default/files/ NORMA_TECNICA_TUBERCULOSIS.pdf.

5.- Sandgren A, Schepisi M S, Sotgiu G, Huitric E, Migliori G B, Manissero D, et al. Tuberculosis transmission between foreign- and native-born populations in the EU/EEA: A systematic review. Eur Respir J 2014; 43(4): 1159-71. doi: 10.1183/09031936.00117213

6.- Ködmön C, Zucs P, van der Werf M J. Migration-related tuberculosis: Epidemiology and characteristics of tuberculosis cases originating outside the European Union and European Economic Area, 2007 to 2013. Eurosurveillance 2016; 21(12): 1-10. doi: 10.2807/1560-7917.

7.- Pareek M, Watson JP, Ormerod LP, Kon OM, Woltmann G, White PJ, et al. Screening of immigrants in the UK for imported latent tuberculosis: A multicentre cohort study and cost-effectiveness analysis. Lancet Infect Dis 2011; 11(6): 435-44. doi: 10.1016/S14733099(11)70069-X

8.- Pareek M, Greenaway C, Noori T, Munoz J, Zenner D, Toms C, et al. The impact of migration on tuberculosis epidemiology and control in high-income countries: a review. BMC Med 2016; 14 (1): 48. doi: 10.1186/ s12916-016-0595-5.

9.- Kamper-Jorgensen Z, Andersen A B, KokJensen A, Kamper-Jorgensen M, Bygbjerg IC, Andersen PH, et al. Migrant tuberculosis: the extent of transmission in a low burden country. BMC Infect Dis 2012; 12: 60. doi: 10.1186/1471-2334-12-60.

10.- Van der Werf M J, Zellweger J P. Impact of migration on tuberculosis epidemiology and control in the EU/EEA. Eurosurveillance. 2016; 21 (12): 8-11. doi: 10.2807/1560-7917. ES.2016.21.12.30174.

11.- Tomás B A, Pell C, Cavanillas A B, Solvas J G, Pool R, Roura M. Tuberculosis in migrant populations. A systematic review of the qualitative literature. PLoS One 2013; 8 (12): 1-12. doi: 10.1371/journal.pone.0082440.

12.- Hayward S, Harding RM, McShane H, Tanner R. Factors influencing the higher incidence of tuberculosis among migrants and ethnic minorities in the UK. F1000Research 2018; 7 (0): 461. doi: 10.12688/f1000research.14476.1

13.- Dhavan P, Dias H M, Creswell J, Weil D. State of the Art Series. An overview of tuberculosis and migration. Int J Tuberc Lung Dis. 2017; 21(6): 610-23. doi: 10.5588/ijtld.16.0917

14.- Abubakar I, Cohen T, Jackson C, Rangaka M, Menzies NA, Hill AN, et al. State of the art. The impact of migration on tuberculosis in the United States. Int J Tuberc Lung Dis. 2018; 22(March): 1392-403. doi: 10.5588/ ijtld.17.0185.

15.- Lönnroth K, Migliori G B, Abubakar I, D’Ambrosio L, De Vries G, Diel R, et al. Towards tuberculosis elimination: An action framework for low-incidence countries. Eur Respir J 2015; 45(4): 928-52. doi: 10.1183/09031936.00214014.

16.- Zuber P, McKenna M, Binkin N, Onorato I, Castro K. Long-term risk of tuberculosis among foreign-born persons in the United States. JAMA 1997; 278(4): 23-30. doi:10.1001/ jama.1997.03550040060038.

17.- Lillebaek T, Andersen A B, Bauer J, Dirksen 
burden country. Respir Res 2018; 19(1): 259.

A, Glismann S, De Haas P, et al. Risk of Mycobacterium tuberculosis transmission in a low-incidence country due to immigration from high-incidence areas. J Clin Microbiol. 2001; 39 (3): 855-61. doi: 10.1128/JCM.39.3.855861.2001.

18.- Abascal E, Pérez-Lago L, Martínez-Lirola M, Chiner-Oms Á, Herranz M, Chaoui I, et al. Whole genome sequencing-based analysis of tuberculosis (TB) in migrants: rapid tools for cross-border surveillance and to distinguish between recent transmission in the host country and new importations. Eurosurveillance. 2019; 24 (4): 1800005. doi: 10.2807/1560-7917. ES.2019.24.4.1800005.

19.- Departamento de Extranjería y Migración. Estadísticas Migratorias 2015. Anu Estadístico. 2017;116.

20.- Instituto Nacional de Estadísticas. Características de la inmigración internacional en Chile, Censo 2017. 2018;

21.- Departamento de Extranjería y Migración del Ministerio del Interior y Seguridad Pública. Migración en Chile 2005-2014. 2014.

22.- Organización Panamericana de la Salud. Informe VII Reunión Regional de países de baja incidencia de tuberculosis de las Américas. 2015;13-4.

23.- Varghese B, Enani M, Alrajhi A, Al Johani S, Albarak A, Althawadi S, et al. Impact of Mycobacterium tuberculosis complex lineages as a determinant of disease phenotypes from an immigrant rich moderate tuberculosis doi: 10.1186/s12931-018-0966-x

24.- Nebenzahl-Guimaraes H, Borgdorff MW, Murray MB, Van Soolingen D. A novel approach - The propensity to propagate (PTP) method for controlling for host factors in studying the transmission of Mycobacterium tuberculosis. PLoS One 2014; 9 (5): 1-8. doi: 10.1371/journal.pone.0097816.

25.- Aguilera XP, Gonzalez C, Najera-De Ferrari M, Hirmas M, Delgado I, Olea A, et al. Tuberculosis in prisoners and their contacts in Chile: Estimating incidence and latent infection. Int J Tuberc Lung Dis 2016; 20(1): 63-70. doi: 10.5588/ijtld.15.0056.

26.- Pan American Health Organization. Tuberculosis in the Americas: Regional report 2015, Epidemiology, Control and Financing. 2016.

27.- WHO. Global Tuberculosis Report 2017. WHO. 2017. 1-262 p.

28.- Joint United Nations Programme on HIV/ AIDS. Ending AIDS progress towards the 9090-90 Targets. Glob Aids Updat. 2017; 198.

29.- Cabieses B, Gálvez P, Ajraz N. Migración internacional y salud: el aporte de las teorías sociales migratorias a las decisiones en salud pública. Rev Peru Med Exp Salud Publica 2018; 35 (2): 285. doi: http://dx.doi. org/10.7440/res54.2015.03.

30.- Bernales M, Cabieses B, McIntyre AM, Chepo M, Flaño J, Obach A. Determinantes sociales de la salud de niños migrantes internacionales en Chile: evidencia cualitativa. Salud Publica Mex 2018; 60 (5, sep-oct): 566. http://dx.doi. org/10.21149/9033.

31.- Chingonzoh R, Manesen MR, Madlavu MJ, Sopiseka N, Nokwe M, Emwerem M, et al. Risk factors for mortality among adults registered on the routine drug resistant tuberculosis reporting database in the Eastern Cape Province, South Africa, 2011 to 2013. PLoS One 2018; 13 (8): 1-15. doi: 10.1371/ journal.pone.0202469.

32.- Gayoso R, Dalcolmo M, Braga JU, Barreira D. Predictors of mortality in multidrugresistant tuberculosis patients from Brazilian reference centers, 2005 to 2012. Brazilian J Infect Dis 2018; 22 (4): 305-10. http://dx.doi. org/10.1016/j.bjid.2018.07.002.

33.- Viana PV de S, Redner P, Ramos J P. Fatores associados ao abandono e ao óbito de casos de tuberculose drogarresistente (TBDR) atendidos em um centro de referência no Rio de Janeiro, Brasil. Cad Saude Publica 2018; 34 (5): 1-11. http://dx.doi.org/10.1590/0102-311x00048217.

34.- Orcau i Palau À, Arcas i Ferré M, Caylà i Buqueras J A, García de Olalla i Rizo P. La tuberculosi a Barcelona. Informe 2014. 2015;65.

35.- Ospina J E, Orcau À, Millet J P, Sánchez F, Casals M, Caylà JA. Community health workers improve contact tracing among immigrants with tuberculosis in Barcelona. BMC Public Health 2012; 12(1): 158. doi: 10.1186/1471-2458-12-158. 